\title{
Mezlocillin in the treatment of gonorrhoea
}

\author{
A. LASSUS AND O-V. RENKONEN
}

From the Department of Dermatology and Venereology, University Central Hospital, Helsinki, and the Department of Serology and Bacteriology, University of Helsinki, Finland

SUMMARY A single, intramuscular dose of $2 \mathrm{~g}$ of mezlocillin was used for the treatment of 158 patients with gonorrhoea. In only three out of 144 patients with urogenital gonorrhoea who were re-examined did the treatment fail. Of 12 patients with pharyngeal gonorrhoea who were examined after treatment 10 were treated successfully with the same dose. The sensitivities of the gonococci to mezlocillin and penicillin $\mathrm{G}$ correlated well, although mezlocillin seemed to be active at lower concentrations on both highly sensitive and less sensitive isolates.

\section{Introduction}

Mezlocillin is a newly developed, semi-synthetic penicillin for parenteral administration. In vitro it has an antibacterial activity encompassing those of the cephalosporins, cephalothin and cephazolin, and the penicillins, ampicillin, carbenicillin, and tircacillin. Its antibacterial spectrum also includes, therefore, Neisseria gonorrhoeae. In vivo mezlocillin is active against certain Gram-negative bacteria, such as Escherichia coli, Klebsiella, Proteus, spp., Pseudomonas aeruginosa, and Haemophilus influenzae. It is also active against enterococci and other Gram-positive bacteria which do not produce $\beta$-lactamase (Metzger, 1976). So far the effect of mezlocillin in the treatment of gonorrhoea has not been reported.

In the present study, $2 \mathrm{~g}$ mezlocillin was used in the treatment of gonorrhoea in men and women.

\section{Patients and methods}

All the 158 patients studied ( 101 men and 57 women) were diagnosed and treated at the outpatient department for venereal diseases at the University Central Hospital, Helsinki, between September 1977 and February 1978.

Specimens for direct microscopy and culture were taken from the urethra in male patients and from the urethra, cervix, and rectal mucosa in female patients before treatment and at each follow-up visit. In addition, specimens for culture were taken from the

Address for reprints: A. Lassus, Department of Dermatology and Venereology, University Central Hospital, Snellmanink 14, Helsinki 17, Finland

Received for publication 7 June 1978 pharynx of 30 male patients and 25 female patients who had had orogenital contact. The male patients were seen once, seven to 14 days after treatment, and the female patients twice, seven to 14 and 14 to 21 days after treatment. The specimens for culture were transported in Stuart's medium to the department of serology and bacteriology, at the University of Helsinki, and plated within eight hours of being collected. In the laboratory the specimens were cultured on two Thayer-Martin media, one of which also contained trimethoprim. Although treatment was given on the basis of positive smear results, only the cases confirmed by culture (including fermentation reactions) were included in the assessment. Sensitivity tests to penicillin G and mezlocillin were carried out by the plate-dilution method described by Reyn et al. (1958).

A freshly prepared solution of $2 \mathrm{~g}$ of mezlocillin in $10 \mathrm{ml}$ of saline was given as an intramuscular injection to both male and female patients.

\section{Results}

The results of the sensitivity tests to mezlocillin and penicillin $G$ are illustrated in the Figure. A clear correlation is evident between the sensitivity of the gonococcal strains to the two antibiotics. Mezlocillin was more active, however, than penicillin on both highly sensitive and less sensitive isolates. All three patients who were considered true treatment failures harboured gonococci which had a decreased sensitivity to both mezlocillin and penicillin G.

Four male patients still had positive culture results from urethral specimens when re-examined (Table 1). One of them admitted to possible reinfection and was treated with a further dose of $2 \mathrm{~g}$ mezlocillin. He had 


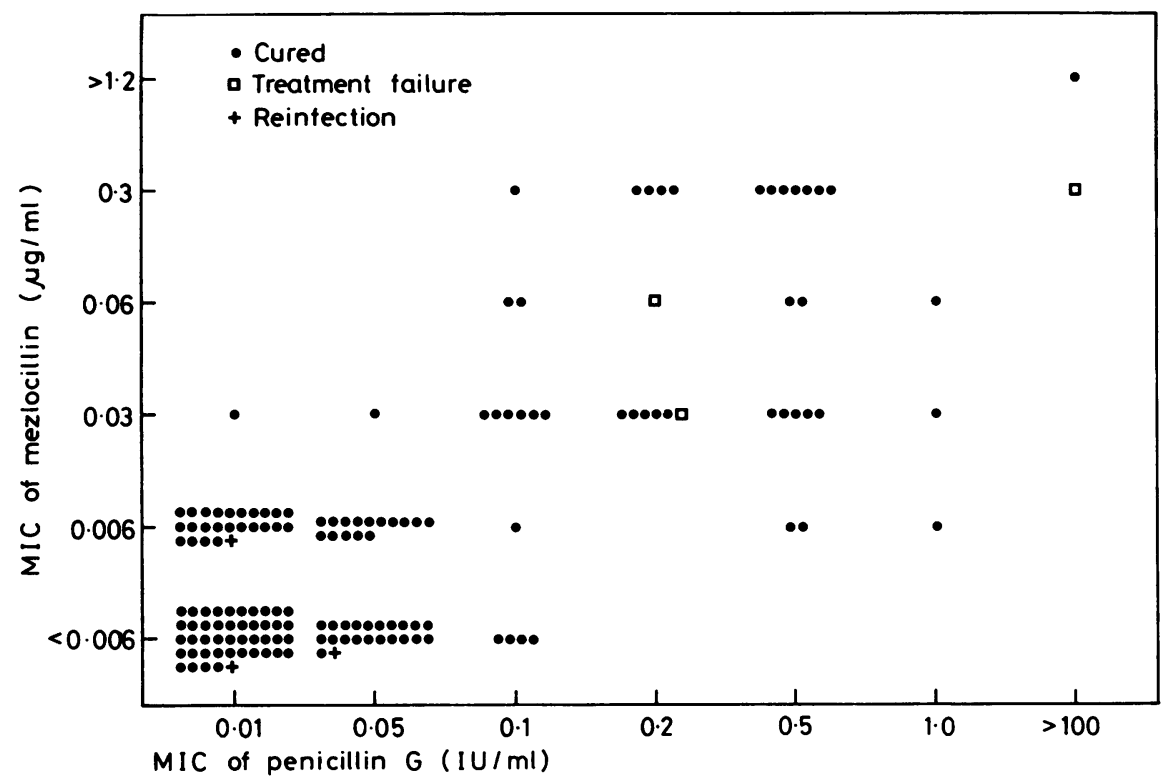

Figure Sensitivity of gonococci to mezlocillin and penicillin $G$ for 158 patients with gonorrhoea. (Three strains were lost in subculture)

Table 1 Results of treatment of urogenital gonorrhoea with mezlocillin

\begin{tabular}{|c|c|c|c|c|c|}
\hline \multirow[b]{2}{*}{ Patients } & \multirow[b]{2}{*}{ Treated } & \multicolumn{2}{|c|}{ Followed up } & \multicolumn{2}{|c|}{$\begin{array}{l}\text { With positive cultures } \\
\text { after treatment }\end{array}$} \\
\hline & & No. & $\%$ & No. & $\begin{array}{l}\% \text { of those } \\
\text { followed up }\end{array}$ \\
\hline $\begin{array}{l}\text { Male } \\
\text { Female } \\
\text { Total }\end{array}$ & $\begin{array}{r}101 \\
57 \\
158\end{array}$ & $\begin{array}{r}92 \\
52 \\
144\end{array}$ & $\begin{array}{l}91 \\
91 \\
91\end{array}$ & $\begin{array}{l}4 \\
2 \\
6\end{array}$ & $\begin{array}{l}4 \\
4 \\
4\end{array}$ \\
\hline
\end{tabular}

a negative culture result when re-examined one week later. In this case the gonococci were initially very sensitive to mezlocillin $(\mathrm{MIC}=0.006 \mu \mathrm{g} / \mathrm{ml}$ ). In the remaining three cases the gonococci showed less sensitivity to mezlocillin (MIC $=0.03,0.06$, and $0.3 \mu \mathrm{g} / \mathrm{ml}$ respectively). The latter strain was of the $\beta$-lactamase-producing type. These three patients were considered to be treatment failures and were all retreated with $2-4 \mathrm{~g}$ of spectinomycin, after which they were cured.

Two female patients had a positive culture result from the cervix when re-examined one at eight days and one at 13 days after treatment; both had gonococci which were sensitive to mezlocillin $(\mathrm{MIC}=0.006 \mu \mathrm{g} / \mathrm{ml})$ and both admitted to possible reinfection. Both patients were cured after further treatment with $2 \mathrm{~g}$ of mezlocillin. A third female patient had gonococci of the $\beta$-lactamase-producing type with an M!C for mezlocillin $>1 \cdot 2 \mu \mathrm{g} / \mathrm{ml}$. She was subsequently treated with spectinomycin $(4 \mathrm{~g})$ despite a negative culture result seven days after she had been treated with mezlocillin. She also had negative culture results when subsequently examined on three occasions.

Seven male patients and seven females had positive gonococcal culture results (supported by sugar fermentation findings) from pharyngeal swabs before treatment. Two of the male patients still had a positive pharyngeal culture result when re-examined one week after mezlocillin treatment (Table 2). All the remaining 10 patients who were re-examined had negative pharyngeal culture results one, two, and three weeks after treatment with mezlocillin.

Mezlocillin was well tolerated in all cases, and none of the patients complained of pain at the injection site.

\section{Discussion}

The present study showed a cure rate of about $98 \%$ for cases of uncomplicated urogenital gonorrhoea with $2 \mathrm{~g}$ of mezlocillin. The cure rate was somewhat higher than the $95-96 \%$ achieved with the routine treatment $(2.4$ megaunits procaine penicillin plus $1 \mathrm{~g}$ probenecid) used at the same clinic. The same dosage of mezlocillin was used in both male and female patients, but the only true relapses occurred in male patients.

The high cure rate in the cases of pharyngeal gonorrhoea was of special interest. Single doses of ampicillin or pivampicillin combined with 
Table 2 Results of treatment of pharyngeal gonorrhoea with mezlocillin

\begin{tabular}{|c|c|c|c|c|c|}
\hline \multirow[b]{2}{*}{ Patients } & \multirow[b]{2}{*}{ Treated } & \multicolumn{2}{|c|}{ Followed up } & \multicolumn{2}{|c|}{$\begin{array}{l}\text { With positive cultures } \\
\text { after treatment }\end{array}$} \\
\hline & & No. & $\%$ & No. & $\begin{array}{l}\% \text { of those } \\
\text { followed up }\end{array}$ \\
\hline Male & 7 & 7 & 100 & 2 & 29 \\
\hline Female & 7 & 5 & 71 & 0 & 0 \\
\hline Total & 14 & 12 & 86 & 2 & 17 \\
\hline
\end{tabular}

probenecid are ineffective in more than $50 \%$ of cases of pharyngeal gonorrhoea (Bro-Jørgensen and Jensen, 1973). The same seems to be true for high intramuscular, single doses of penicillin G. Mezlocillin seems worth trying for the treatment of larger series of patients with pharyngeal gonorrhoea.
The sensitivity of the gonococci to mezlocillin and penicillin $G$ correlated well, although mezlocillin seemed to be active at lower concentrations on both highly sensitive and less sensitive isolates. Crossresistance could be expected as both antibiotics are penicillins.

Thus a single dose of $2 \mathrm{~g}$ of mezlocillin is highly effective in the treatment of uncomplicated urogenital gonorrhoea and has a promising effect on cases of pharyngeal gonorrhoea.

\section{References}

Bro-Jørgensen, A. and Jensen, T. (1973). Gonococcal pharyngeal infections. Report of 110 cases. British Journal of Venereal Diseases, 49, 491-499.

Metzger, K. (1976). The activity of mezlocillin (BAY $\mathrm{f} 1353$ ) in vitro and in vivo. Symposium of Future Trends in Chemotherapy, Tirrania/Pisa, 16 May 1976. 